\title{
ИСПОЛЬЗОВАНИЕ МИКРОПРОЦЕССОРНЫХ КОНТРОЛЛЕРОВ И ПРОГРАММ УКРАИНСКОГО ПРОИЗВОДСТВА ДЛЯ МОДЕРНИЗАЦИИ СИСТЕМЫ УПРАВЛЕНИЯ ДИФФУЗИОННОГО ОТДЕЛЕНИЯ САХАРНОГО ЗАВОДА
}

Use of microprocessors-based comptrollers and programs of the Ukrainian production for modernisation of the control system of sugar-house extraction station

Скаковский Ю.М. ${ }^{1}$, Бабков А.B. ${ }^{1}$ (Skakovsky Y.M. Babkov A.V.)

${ }^{1}$ Одеская национальная академия пищевых технологий, Одесса

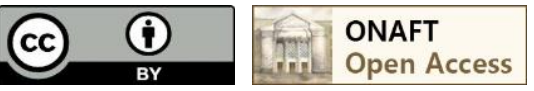

DOI: $10.15673 /$

\begin{abstract}
Аннотация
Рассматривается комплекс технических решений по модернизации управляющей системы станции извлечения сока сахарного завода. Система проектируется на базе микропроцессорных контроллеров и программ. Имитационное моделирование проводилось на специализированом стенде с применением промышленных контроллеров и программ Украинского производства. Результаты исследований применяются для модернизации управляющей системы сахарного завода.
\end{abstract}

Abstract

The complex of technical decisions for modernisation of control system of the sugar-house extraction station is considered. The system is designed with microprocessors-based comptrollers and programs. An imitation design was conducted on the special stand with the use of industrial comptrollers and programs of the Ukrainian production. Research results are applied for modernisation of control system of the sugar-house.

Ключевые слова

Система автоматического управления, автоматизированное рабочее место оператора, микропроцессорный контроллер, диффузионное отделение, сахарный завод.

\section{Введение}

В Одесской национальной академии пищевых технологий в рамках хоздоговорной тематики выполняются работы по оказанию научно-технической помощи при модернизации систем автоматизации для повышения эффективности их функционирования на Красиловском свеклосахарном заводе (Хмельницкой области). Создана многоуровневая АСУТП свеклосахарного производства, включающая автоматизированные рабочие места (АРМ) операторов основных технологических участков, АРМы диспетчера, главного технолога и директора на базе микропроцессорных контроллеров, компьютеров и локальной сети на базе технологии Ethernet [1]. В настоящее время выполнены работы по модернизации систем автоматизации диффузионного отделения, включающего диффузионную установку колонного типа.

Основные технические решения. В составе АСУТП диффузионного отделения помимо традиционных задач контроля и регулирования регламентированных переменных реализована подсистема оперативного учета, вычисления и представления основных технико-экономических показателей работы отделения [1, 2], которая 
обеспечивает представление результатов расчетов на различных уровнях управления (АРМах) для диспетчера (начальника смены), главного инженера, главного технолога, директора и т.п.

Диффузионное отделение является основным с точки зрения формирования материального потока сахарного завода, ритмичность его работы во многом определяет ритмичность всего сахарного производства.

Традиционные критерии управления заводом, принятые в производстве сахара, при их наглядной интерпретации могут быть полезными не только при анализе успешности функционирования предприятия за сутки, декаду, месяц, но и при текущем анализе внутрисменной работы. При необходимости, экранные формы, таблицы, графики и т. П., могут быть выведены на принтер и сведены в архивы бумажных документов.

Представляемые данные используются техническими руководителями завода для оперативного контроля, анализа и своевременного принятия решений по управлению технологическими процессами свеклосахарного производства (корректировки режимов, производительности и т. п.), оценки работы отдельных смен и завода в целом.

Перечень задач, реализованных в составе АСУТП диффузионного отделения, включает:

- $\quad$ контроль технологических переменных и состояния оборудования (привода, сита);

- регулирование:

- стабилизация:

- уровней: в дифаппарате; в шахте ошпаривателя; в мешалке ошпаривателя; в сборнике воды;

- температур: сокостружечной смеси; питательной воды;

- $\quad$ соотношений (следящее регулирование): «расходов дифсока - стружки»; «расходов сока рециркуляции стружки»;

- $\quad$ оперативный учет стружки (сырья);

- вычисление показателей работы: коэффициент откачки (Котк), коэффициент соотношения Кс.рец.-стр., коэффициент неравномерности работы (текущий, интегр.);

- «ПЛАН-ФАКТ» и др.

Выбор линии микропроцессорных устройства производился с учетом реальных возможностей персонала службы главного метролога по освоению и обслуживанию подобной техники. АСУТП на этом предприятии реализовано на базе функционально-программируемых контроллеров отечественного производства и компьютеров, оснащенных SCADA-системой «ИНДЕЛ», разработанной полтавским предприятием «ИНФОТЕХПРОМ». Набор драйверов, поставляемых в комплекте со SCADA-системой, обеспечивает обмен информацией с контроллерами, как отечественного производства Ремиконт (110, 130), МИК (предприятие «МИКРОЛ», г. Ивано-Франковск), так и импортного производства (Siemens , VIPA и др.). Предпочтение было отдано программируемым контроллерам предприятия «МИКРОЛ» МИК-52, микропроцессорным регуляторам МИК-12, МТР-8, исходя из критерия «стоимость - качество», с учетом близости программного обеспечения для этого программируемого контроллера (редактор «ф») к знакомому персоналу службы КИП и А редактору для функционирующих на предприятии микропроцессорных контроллеров (МПК).

Взамен использованнного ранее стационарного компьютера, установленного в операторском пункте, был применен неттоп типа 3QNTP-D2560, отличающийся миниатюрными размерами корпуса, что позволяет крепить его на задною стенку монитора, гальванически развязанным питанием (блок питания входит в комплект поставки), развитым набором интерфейсных устройств связи, включая порт Ethernet, USB и др. Установленная операционная система WINDOWS-XP обеспечивает реализацию всех функций SCADA-системы «ИНДЕЛ». Выбор последней, вместо предлагаемой производителями контроллеров SCADA-системы «Visual Intellect», обусловлен прежде всего простотой и удобством работы по настройке драйвера MODBUS RTU, входящего в комплект поставки SCADAсистемы «ИНДЕЛ», позволяющего обеспечить связь со всеми контроллерами фирмы «МИКРОЛ» и достаточно развитыми функциональными возможностями, хорошо знакомыми как операторам так и техническим руководителям предприятия, чьи рабочие места оснащены АРМ.

На рис. 1 приведена основная экранная форма АРМ оператора диффузионного отделения, где помимо информации о режимных параметрах и состоянии оборудования приведены результаты оперативного почасового учета расхода стружки, поступающей в дифаппарат, а также табло «ПЛАН-ФАКТ». Кроме того, отражены результаты вычисления показателей работы: коэффициенты откачки (К соотношения «стружка-вода». Указанные результаты наглядно показывают ритмичность работы участка в течение смены, и, наряду с графическими представлениями текущего и интегрированного коэффициента неритмичности работы, позволяют проводить диспетчеру объективный анализ работы и, при необходимости, своевременно вносить коррективы.

Оценки почасовой переработки сырья (стружки), выполняются путем интегрирования сигнала его мгновенного расхода, поступающего в контроллер от ленточных весов, в пределах текущего часа смены: 


\section{ТЕХНІЧНІ ЗАСОБИ ТА ІНФОРМАЦЙНІ ТЕХНОЛОГІЇ В СИСТЕМАХ УПРАВЛІННЯ}

$$
Q_{i+1}=\int_{t_{i}}^{t_{i+1}} F_{c}(t) d t
$$

где $F_{c}(t)-$ мгновенный расход стружки, т/ч;

$t_{i}$ - момент времени начала очередного часа, ч;

$i$ - порядковый номер часа $(i=0, \ldots, 11)$, соответствует 12-ти часовой продолжительности смены; $Q_{i+1}$ - количество стружки, переработанной за $i$-й час.

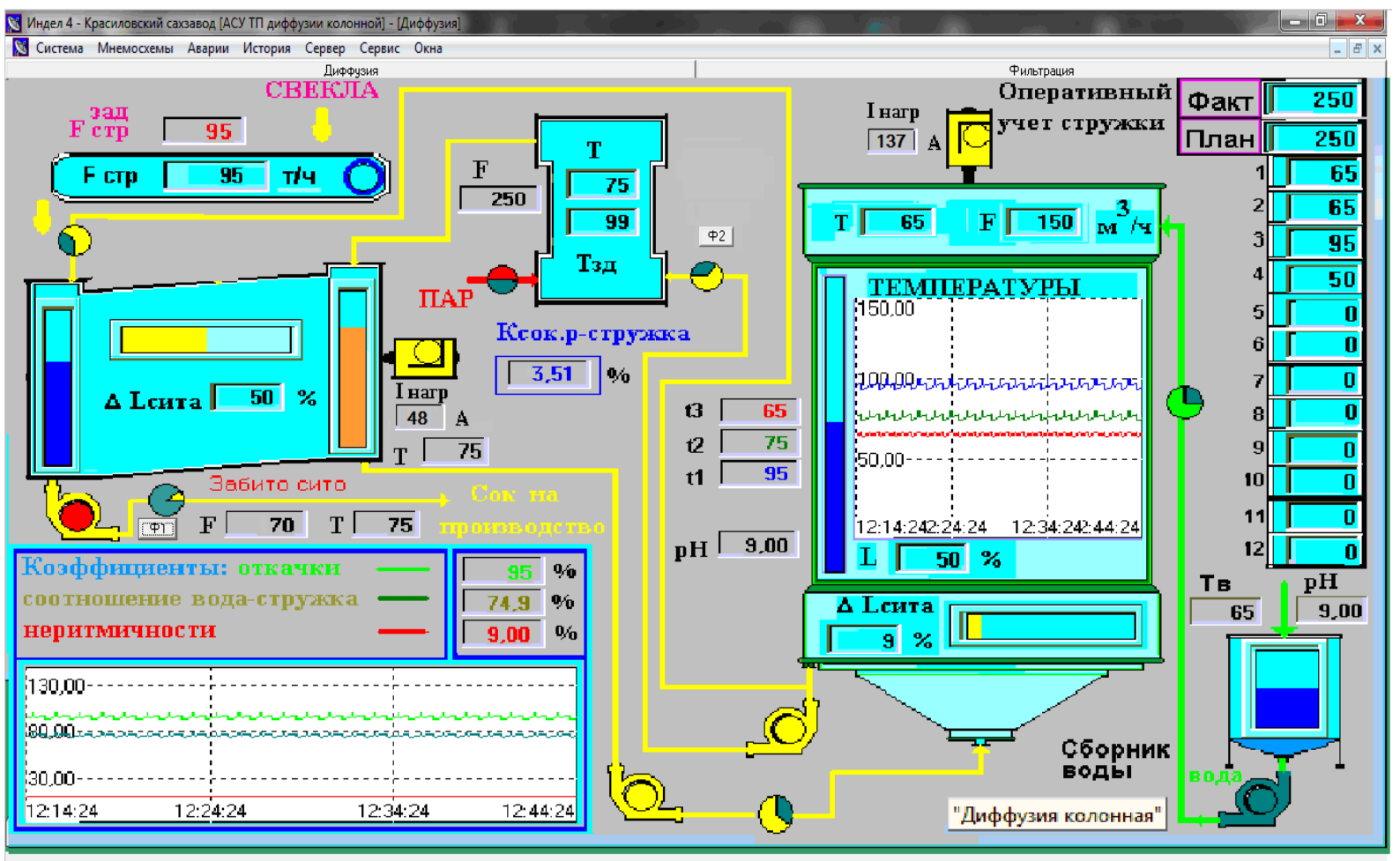

Рис. 1 Экранная форма АРМ оператора диффузии (имитатор)

Лабораторный имитационный стенд, на котором проводились исследования, содержал: МПК МИК-52, МТР8, МИК-12, аналоговые задатчики РЗД-22 (с токовым выходом 4,..,20 мА), блок преобразования интерфейсов БПИ-52, компьютер с установленным программным обеспечением: драйвер для БПИ-52, редактор «а», SCADAсистема «ИНДЕЛ» с драйвером MODBUS RTU, обеспечивающим обмен с МИК-52, МТР-8, МИК-12 по двухпроводному интерфейсу RS-485.

Программную реализацию алгоритмов оперативного учета и вычисления показателей было решено выполнять в программируемом контроллере МИК-52, что существенно повышает точность полученных результатов, поскольку опыт эксплуатации подобных систем показывает, что показатели надежности функционирования данного контроллера выше аналогичных показателей надежности функционирования компьютера (неттопа). Последнее обстоятельство особенно важно в связи с реализацией функции интегрирования в расчетных задачах. На рис. 2 представлен фрагмент программы, реализующей задачи оперативного учета сырья, оценивания показателей и управления на языке $\alpha$ для контроллера МИК-52.

В программе алгоблоки (АБ) аналогового ввода $\mathrm{AIN}(05)$ 3, 34-39, используются для ввода и предварительной обработки сигналов от датчиков и внешних задатчиков; АБ дискретного ввода DIN(06) 15 служит для внешнего управления программой, позволяя при необходимости приостанавливать работу таймера АБ TIMER(38) 6 либо осуществлять перезапуск программы. Аналогичную функцию выполняют АБ dSET(83) 4, при управлении программой с дополнительной экранной формы Ф1 (рис. 3), позволяющей настраивать контур регулирования соотношения «СОК - СТРУЖКА» с экрана. Сигнал от АБ $\mathrm{AIN}(05) 3$ (расход стружки от ленточных весов) поступает на интеграторы АБ INTEGR(17) 1-12, по результатам интегрирования формируются почасовые оценки количества переработанного сырья (стружки) с помощью сумматоров АБ SUMM(13). Для управления интеграторами служат АБ STEP(85) 10, 11, обеспечивая своевременный запуск и остановку каждого интегратора. 


\section{ТЕХНІЧНІ ЗАСОБИ ТА ІНФОРМАЦІЙНІ ТЕХНОЛОГІЇ В СИСТЕМАХ УПРАВЛІННЯ}

Решение задачи «ПЛАН - ФАКТ» и оценивания интегрированного коэффициента неритмичности (К обеспечивают АБ INTEGR(17) 44, 49, а текущих значений К

Контур регулирования соотношения «СОК - СТРУЖКА» (следящий регулятор) реализован в АБ $\mathrm{PID}(60) 41$ с использованием сигналов от АБ $\mathrm{AIN}(05)$ 3, 34, 35 и АБ умножения MUL(12) 40. Управление расходом сока, отбираемого с колонной диффузии осуществляется с помощью частотного преобразователя, на вход которого поступает сигнал с АБ АОТ(08) 42. Для окончательного представления данных в экранных формах рис. 2 , 3 полученные результаты масштабируются либо с помощью настроек программ МПК, либо непосредственно в SCADA-системе.



Рис. 2 - Фрагмент программы, реализующей задачи оперативного учета, вычисления показателей и управления на языке $\alpha$ для контроллера МИК-52 
Вспомогательные экранные формы (Ф1, Ф2), спроектированные для наладки «сложных» контуров регулирования доступны только уполномоченным специалистам КИП и А («запаролены»).

Вся сеть контроллеров, спроектированная для реализации АРМ оператора диффузионного отделения, состоит из 11-ти МПК, в том числе 3-х МИК-52, подключенных к компьютеру через преобразователь БПИ-52 (RS485 USB). Все контроллеры щитового исполнения, что позволяет использовать их в качестве показывающих приборов и органов дистанционного управления при необходимости перехода в дистанционный режим. Питание электроэнергией основных элементов АРМ оператора осуществляется через источник бесперебойного питания. АРМ оператора, включая щит контроля и управления расположен в специализированном помещении операторском пункте, где для создания комфортных условий обеспечена приточная вентиляция.

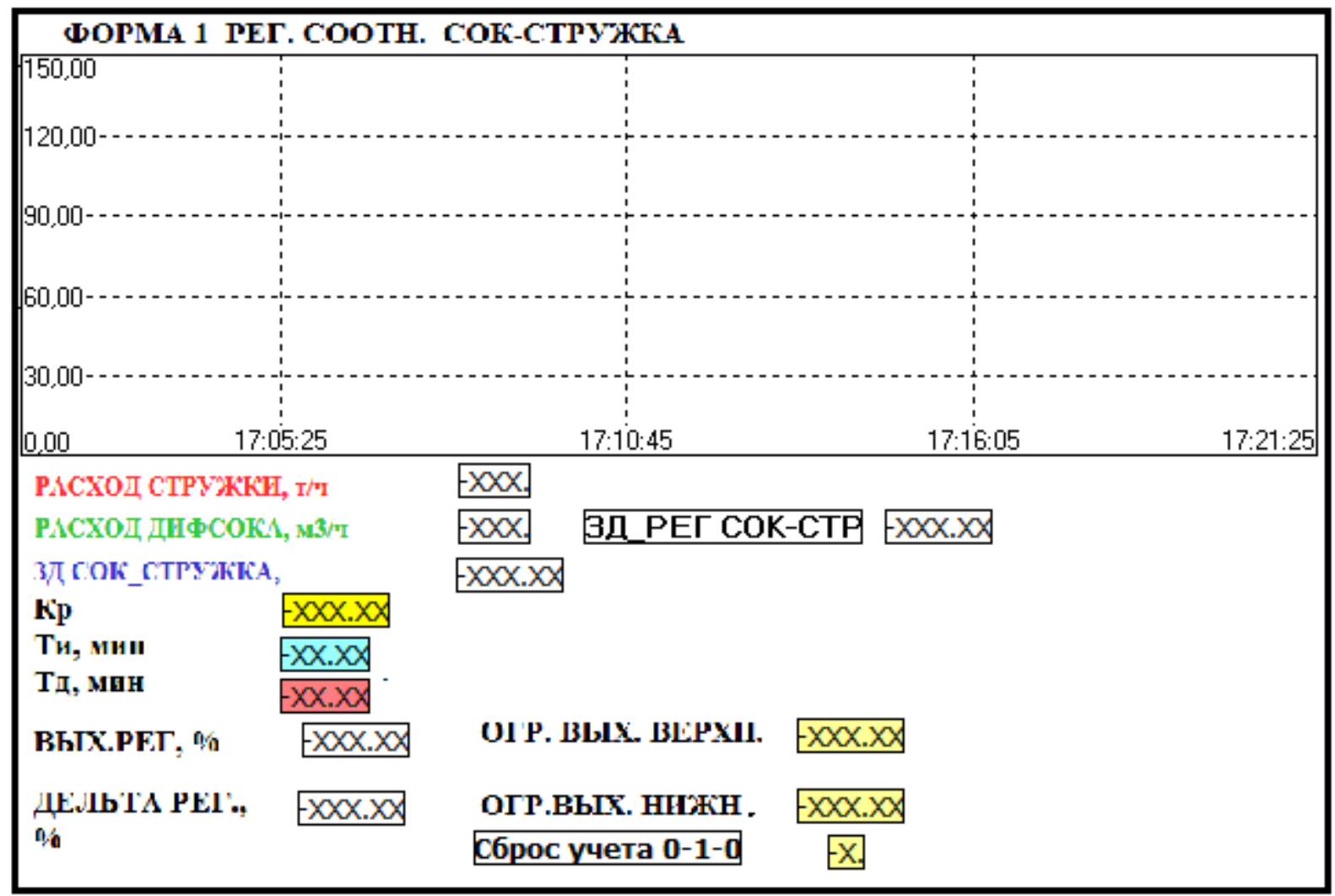

Рис. 3 - Вспомогательная экранная форма наладчика контура регулирования соотношения «СОК-СТРУЖКА»

Модернизированная АСУТП диффузионного отделения Красиловского сахарного завода сдана в промышленную эксплуатацию и функционирует два сезона (2013/2014 гг., 2014/2015 гг.).

Опыт ее эксплуатации подтвердил рациональность принятых по результатам проведеных предпроектных исследований технических решений, которые обеспечили достижение поставленных целей, удобство для оперативного персонала и адаптацию данной АСУТП в структуру многоуровневой АСУТП завода.

\section{Выводы}

Проведенные предпроектные исследования с использованием методов имитационного моделирования на специализированном стенде, оборудованном промышленными контроллерами, позволили разработать комплекс технических решений, обеспечивающих успешную эксплуатацию модернизированной АСУТП.

Технические и программные средства Украинского производства обеспечивают достаточно комфортные условия, как разработчикам систем управления, так и оперативному персоналу предприятия.

Выполненный комплекс работ позволяет сделать вывод о целесообразности продолжения работ по модернизации систем управления предприятия, используя подобные методы и подходы к принятию основных технических решений. 
Литература

1. Жуковский Э.И. Реализация задачи оценивания показателей работы завода в АСУТП сахарного производства/ Э.И. Жуковский, Ю.М. Скаковский, В.Д. Витвицкий, А.В. Бабков // Автоматизация технологических и бизнес-процессов. - Одесса: 2010 - №3.- С.16-26.;

2. Вітвіцький В.Д. Рішення задач оперативного обліку в АСУТП ділянок цукробурякового виробництва/ В.Д. Вітвіцький, Ю.М. Скаковський // Наук. пр. Одес. нац. акад. харч. техн./ Міністерство освіти і науки України. - Одеса: 2004 -Вип. 27.- С.213-221.

Referens

1. Gukovskiy E.Y. Realizacia zadachi ocenivania pokazateley raboti zavoda v ASUTP saharnogo proizvodstva/ E.Y. Gukovskiy, Y.M. Skakovskiy, V.D. Vitvitskiy, A.V. Babkov //Avtomatizacia tehnologicheskih i biznesprocesov.- Odessa:2010 - №3.- S.16-26.;

2. Vitvitskiy V.D. Rishennia zadach operativnogo obliku v ASUTP dilianok cukroburiakovogo virobnictva/ V.D. Vitvitskiy, Y.M. Skakovskiy //Nauk. pr. ONAHT/Ministerstvo osviti Ukraini.- Odesa: 2004.- Vip.27.- S.213221.

\title{
РАЗРАБОТКА МОДЕЛИ И ПРОГРАММНЫХ СРЕДСТВ ДЛЯ СОЗДАНИЯ РОБАСТНОЙ СИСТЕМЫ УПРАВЛЕНИЯ ТЕПЛООБМЕННИКОМ
}

Development of the model and software for heat exchanger robust control system design

Стопакевич A. O. ${ }^{1}$ (Stopakevych A. O.)

${ }^{1}$ Одесская национальная академия связи им. Попова, Одесса, E-mail: stopakevich@gmail.com

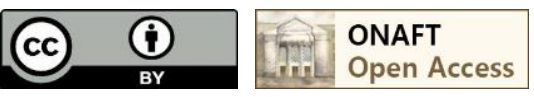

DOI: $10.15673 /$

\begin{abstract}
Введение
В статье рассматривается построение математической модели, а также структура математического и программного обеспечения для синтеза робастных систем автоматического управления парожидкостными теплообменниками. Показана актуальность задачи и описаны трудности её решения. Отличием предложенного алгоритма построения модели теплообменника является минимальное число требуемых исходных данных: начальная и конечная температуры жидкости, массовый расход жидкости и давление в паропроводе. Алгоритмизируется конструктивный расчет, расчет потерь, расчет регулирующего органа, расчет модели динамики теплообменника, расчет модели динамики датчика температуры, расчет регулятора с учетом робастности замкнутой системы автоматического управления. Алгоритм конструктивного расчета позволяет выбрать стандартный теплообменник. Программное обеспечение реализует разработанные алгоритмы. Анализ переходных процессов, полученных при ряде нагрузок теплообменника, доказывает, что разработанная система автоматического управления является робастной.
\end{abstract}

\section{Abstract}

The article describes the development of a mathematical model, mathware and software for steam-liquid heat exchanger robust control system design. The article spotlights the problem actuality and difficulties of its solving. The advantage of proposed algorithm for the exchanger model design is a minimal number of input variables, namely initial and final temperature of the liquid, mass flow rate of the liquid, pressure in the steam line. The algorithm includes constructive design, calculation of losses, calculation of the control valve, calculation of the heat exchanger dynamic model, calculation of the temperature sensor dynamic model and design of robust controller for the closed-loop control system. The algorithm of constructive design allows choosing a heat exchanger from 